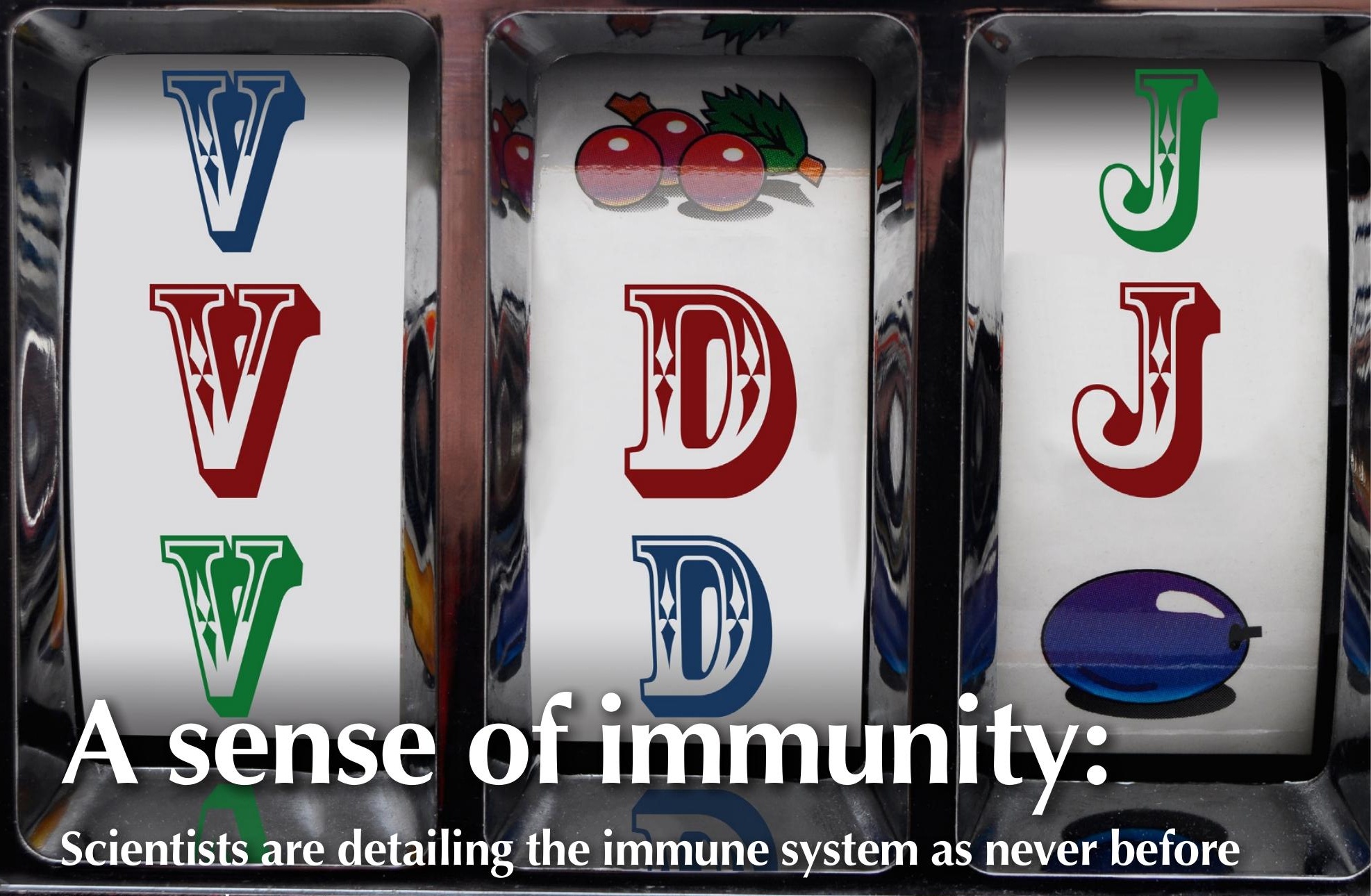

By Amanda B. Keener

By the end of this winter, as many as 150 million people in the US may have received this season's flu vaccine, but not all of them will be protected from catching the flu. In fact, up to $40 \%$ of people over the age of 60 won't even make antibodies in responses to the vaccine ${ }^{1}$. Immunologist Mark Davis is trying to understand why. For more than seven years, he and other researchers at Stanford's Human Immune Monitoring Center (HIMC) in Palo Alto, California, have been taking blood samples from about 100 volunteers to find out how immune cell responses to flu vaccination vary among different age groups.

The Stanford researchers are looking for new ways to define immune health and predict successful responses to vaccines. "The only surrogate marker we've had for vaccine development has been antibody responses, and antibody responses are not that important for a lot of diseases," Davis says. Antibodies against $\mathrm{HIV}$, for example, are not protective unless they bind and neutralize many strains of the virus, and some formulations of the malaria vaccine candidate, RTS,S, induce antibodies but do not provide protection ${ }^{2}$

Davis and his team are instead looking for surrogate markers of vaccine efficacy within cells whose activation precedes antibody production. These cells that make up the adaptive immune system, $\mathrm{T}$ and $\mathrm{B}$ cells, exist within individuals in unique combinations called repertoires, which shape how the immune system interacts with its environment. Repertoire composition is thought to influence whether someone develops an autoimmune disease such as multiple sclerosis or mounts a robust response to a tetanus vaccine.

Each $\mathrm{B}$ or $\mathrm{T}$ cell carries its own unique receptor, which is basically an antenna designed to bind and detect specific foreign substances that enter the body, such as bacteria. What that 'antenna' looks like depends on its parts, which are formed by stretches of DNA known as variable, diversity and joining gene segmentsor VDJ segments-which can recombine in a random and unpredictable process that results in a huge degree of diversity among the cells.

Thanks to mutations that get introduced as the segments recombine, there are at least 10 trillion different ways in which they could come together to make a receptor ${ }^{3,4}$. Although the human body contains far fewer than 10 trillion B or T cells, the diversity among the cells within any one individual, and between the repertoires in any two individuals (even identical twins) is still large enough to make it very difficult to catalog and track how different receptors influence human health. "We know a lot about [B and T cells] in terms of basic biology," says Davis, "but we don't know a lot about them regarding human disease. That has required new technologies."

That technology has arrived, and already it is being employed. In November, two companies announced new ways to put it to use. The San Francisco-based biotechnology company Sequenta said it would seek approval from the US Food and Drug Administration for a clinical kit to track cancer cells in treated lymphoma patients on the basis of receptor sequences. Meanwhile, Seattle-based Adaptive Biotechnologies began marketing a standardized assay for researchers to catalog the T cell repertoire of human samples. As these technologies are deployed and further improved upon, the human immune system will become less of a black box, and details about how $\mathrm{B}$ and $\mathrm{T}$ cells differ among people could help reveal patterns displayed by immune responses that best protect against specific infections.

\section{True diversity}

An immune cell might start out as a tabula rasa, but once a young $\mathrm{B}$ or $\mathrm{T}$ cell's receptor arrangement is determined, it leaves its place of maturation (the bone marrow for B cells and the thymus for $\mathrm{T}$ cells) and produces copies of itself, which are thus called clones. Notably, the genetic sequence of a small but unique 


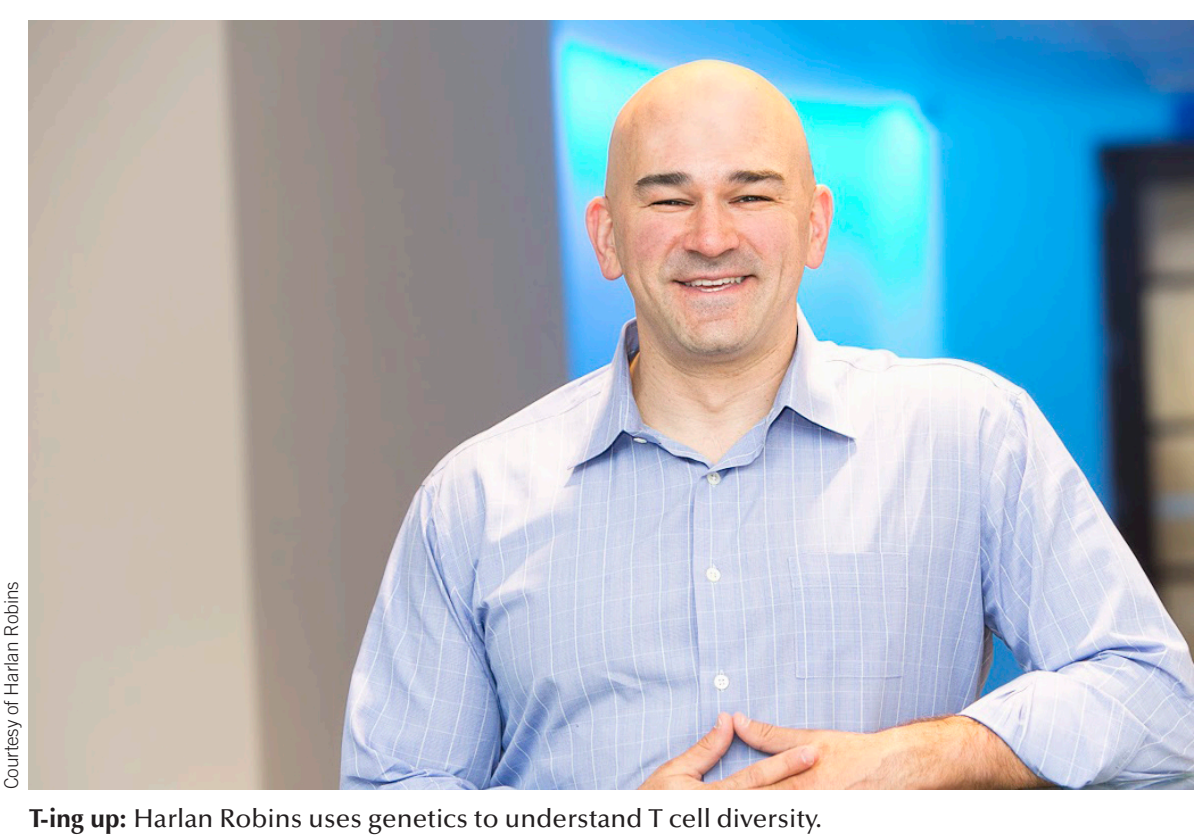

region of a $\mathrm{B}$ or $\mathrm{T}$ cell receptor is enough to give the cell its distinct clonal identity. Until a decade ago, sequencing a single one of these regions cost an average of $\$ 5-\$ 10$, thus limiting immunologists to hundreds or thousands of sequences per experiment when they really needed to generate millions. These restrictions meant scientists could only ever get "a glimpse of the true diversity," says Robert Holt, head of the Michael Smith Genome Sciences Center at the British Columbia Cancer Agency in Vancouver.

Holt was among the first to tackle immunological questions with next-generation sequencing, which made it possible to read out millions of sequence strands at the same time. By using this technology, Holt's group reported sequences for over 30,000 unique T cell receptors from hundreds of pooled samples of human blood ${ }^{5}$. Before that, a total of only about 3,000 T cell receptors had ever been identified. Almost simultaneously, 100 miles south, at the Fred Hutchinson Cancer Research Center in Seattle, computational biologist Harlan Robins was working with clinical immunologists and geneticists to characterize the diversity of T cells each person carries.

"Now that it's possible to get this data, it really expands the types of questions you can ask," says Stanford School of Medicine pathologist Scott Boyd, who led some of the earliest work on normal human B cell repertoires with geneticist Andrew Fire ${ }^{6}$. The two recently used next-generation sequencing to describe unique qualities of the rare B cells that make broadly neutralizing antibodies in HIV infection ${ }^{7}$. Boyd has also been studying the HIMC aging cohort, which has so far shown that repertoire diversity seems to drop with age ${ }^{8,9}$.
Both Boyd and Robins saw early on that the relative abundance of specific $B$ and $T$ cell clones could serve as a clinical biomarker during disease or after vaccination. In lymphomas the rearranged $\mathrm{B}$ or $\mathrm{T}$ cell receptor serves as a tumor-specific marker. This concept became the basis for companies such as Sequenta and Adaptive Biotechnologies to offer sequencebased testing for cancer cells present in the blood or bone marrow of lymphoma patients during apparent clinical remission. The presence of these cancerous cells, known as minimal residual disease, forms a measure that doctors use to predict relapse. In a 2012 study, scientists, including those at Adaptive, showed that this technique was more sensitive than the standard cell counting-based blood test and could detect minimal residual disease in half of the lymphoma patients who tested negative by the older method ${ }^{10}$.

\section{The other half}

Although the potential applications for nextgeneration sequencing in immunology seem boundless, it has so far only been able to reveal part of the picture. "The majority of experiments going on right now are sequencing one thing at a time," Boyd says. B and T cell receptors are made of two genes, and most repertoire analyses so far have focused on the larger of the two: the heavy chain in the case of B cells and the beta chain of T cells. That's a problem, Davis explains. "If you just get a list of all the beta chains, that tells you something about the composition, but it doesn't allow you to reconstruct the T cell receptor," he says.

Sequencing both chains of each individual receptor is necessary to find out precisely what piece of a pathogen or vaccine, called an antigen, a T or B cell is 'seeing', or binding. Genetic material from single cells has to be kept separate to keep track of which genes came from which cell, and that limits the number of cells that can be analyzed in a given experiment to the order of hundreds. That may be enough to study immediate responses to a known entity, such as the flu vaccine, because flu-specific cells will divide and dominate the repertoire and flu-binding cells can be preselected before sequencing. Robins notes that these techniques don't help if the antigen is completely unknown; you could never know the identity of every randomly mutated cancer cell protein recognized by the $\mathrm{T}$ cells attacking a tumor. "At this point it's an unsolved problem," Robins says. "It's sort of the holy grail of the field."

\section{Making sense of the data}

Among those making significant progress toward the holy grail of sequencing single cells in larger numbers, George Georgiou, a biomedical engineer at the University of Texas at Austin, has developed a method for breaking open individual cells in tiny wells and attaching the two receptor chain genes together before sequencing ${ }^{11}$. He is also working on an alternate technique that uses lipid spheres instead of wells, which will dramatically speed up the process $^{12}$. Georgiou is a scientific advisor for the biomedical startup AbVitro, which plans to use these new technologies to identify drug targets and develop new monoclonal antibody-based therapies.

Even as he works to make the sequencing process more efficient, Georgiou stresses the importance of incorporating B cell receptor sequences into a bigger picture of the overall immune response. "Sequences tell you a small part of the story," he says. He is currently developing ways to link the $\mathrm{B}$ cell receptor repertoire to the antibody repertoire in the blood, because the ultimate function of a $\mathrm{B}$ cell is to make antibody.

"If we had a better understanding of what a particular vaccine did in terms of the whole immune response-and that's B cells, T cells, innate immunity-then we could make better choices and speed things up a lot," says Davis, who has recently developed techniques to simultaneously examine $\mathrm{T}$ cell receptor sequences and $\mathrm{T}$ cell function ${ }^{11}$. Jamie Scott, a molecular immunologist at Simon Fraser University in Burnaby, British Columbia agrees, saying that when it comes to vaccine responses, "We are like a bunch of cavemen with our hands dragging on the ground."

The desire for a more comprehensive understanding of immune responses has led 


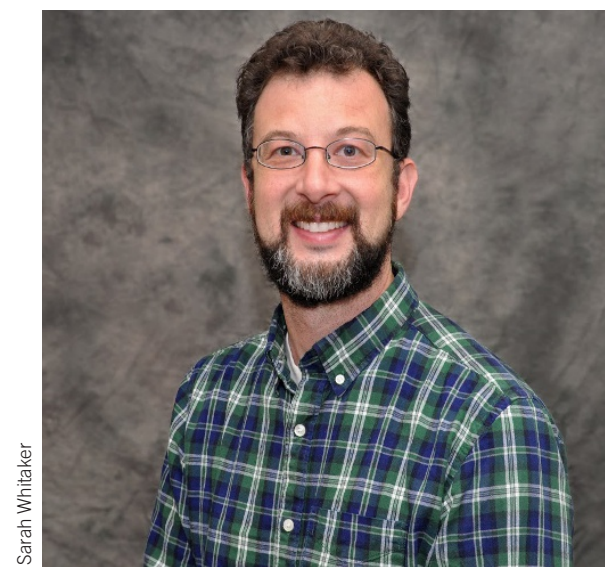

Receptor interceptor: Kleinstein traces sequences.

to initiatives such as the Human Immunology Project Consortium (HIPC), which has been funded by the National Institute of Allergy and Infectious Disease (NIAID) to bring multiple types of immunological data from seven institutions together in one public database (announced in Nat. Biotech. 32, 146-148 2014). "It's something that each of the centers individually has a hard time balancing," says Shai Shen-Orr, who studies systems immunology at the Technion-Israel Institute of Technology and is also part of HIPC.

Currently, HIPC researchers are pulling together data about the immune systems of 600 individuals in the US before and after flu vaccination; this data will help predict who will and won't respond to the shot. Much of the consortium's effort has been focused on flu because a lot is already known about how it affects immune cells. "It's been a very good proving ground for new technologies," says Davis, who serves on the HIPC steering committee. HIPC researchers are also studying malaria and yellow fever vaccine responses, immunity in infants and pregnant women, and immune signatures of several viral and bacterial infections.

HIPC will soon launch an online analysis platform called ImmuneSpace and will drop its data onto the NIAID's larger Immunology Database and Analysis Portal (ImmPort). However, neither initiative fully incorporates repertoire sequence data sets. "People are only now realizing how huge these data sets are and how difficult it is to compare different research from other labs," says Felix Breden, a population geneticist who is married to Scott and who also works at Simon Fraser. To facilitate more streamlined meta-analysis, the pair wants to glean human adaptive receptor sequence data into a publicly accessible database they are calling iReceptor.

With support from the Canadian Institute of Health Research, Breden and Scott hosted a meeting in September involving representatives from HIPC, ImmPort, Genmab and Novartis, as well as several scientists, including Georgiou and Holt. The attendees formed an organizing committee and developed a set of questions about data management and quality-control standards that they will address at a larger meeting to be held at Simon Fraser University in May. The group will also devise plans to integrate iReceptor with currently available sequence analysis tools, such asVDJServer, which was slated to launch in December as Nature Medicine went to press. VDJServer will give researchers the option to selectively or publicly share their data, and provide software that immunologists without bioinformatics training can use to ask basic questions about diversity and clone frequencies.

Steven Kleinstein, a computational immunologist at Yale University School of Medicine who developed some of this software, hopes that VDJServer will eventually facilitate more complex analyses, like building family trees of B cells and T cells on the basis of their receptor sequences. His group recently used this sort of analysis to trace the origin of braindamaging B cells in four multiple sclerosis patients back to the cervical lymph nodes, shifting the previous view that the cells mainly expand within the brain ${ }^{14}$.

\section{Disease progression}

Although Kleinstein sees great potential for repertoire analysis to improve understanding of how diseases progress, some would rather use complex immunologic data to catch diseases before they set in. "Often by the time we see patients with fulminant disease, they have an overactive immune system. What you really want to know is before that occurred, what one or two things changed," says Dennis Ausiello, who co-founded the Center for Assessment Technology and Continuous Health (CATCH), which integrates research from Boston's Massachusetts General Hospital, where Ausiello serves as the chair of medicine, and from the nearby Massachusetts Institute of Technology. The goal of CATCH is to find biomarkers that can signal changes in patient health on a continuous basis.

"We are pretty naive about the status of the immune system in almost all diseases," says Ausiello. CATCH currently tracks things like blood pressure and glucose levels, but he says the ability to merge these so-called "blunt instruments" with immunological information is coming. An early example is a nanotube patch that can sample innate immune cells called macrophages in the skin.

Adaptive Biotechnologies is working to characterize a healthy adaptive repertoire outside of the context of vaccination or disease as a sort of reference point. "What we'd like to really get at is to truly define immunocompetence," says Robins. Both Adaptive and $\mathrm{CATCH}$ have been discussing potential collaborations with Google X's Baseline project, which seeks to define human health from every possible angle. The Pasteur Institute's Milieu Interieur project-named after the $19^{\text {th }}$ century French physiologist Claude Bernard's concept that a fluid environment maintains stability for organs and tissues-is examining samples from 1,000 healthy volunteers to define the makeup of a healthy immune system. Likewise, the Human Functional Genomics Project, a sequencing effort based in the Netherlands, is investigating aspects of human immunity.

For now, immune repertoire health remains outside the scope of the standard doctor's office checkup. In the meantime, small clinical studies are showing that details about immune cells could become a way to stratify patients or predict who will and won't respond to certain treatments. Repertoire diversity could potentially predict the success of stem cell transplants ${ }^{15,16}$ and improve treatment for graftversus-host disease ${ }^{17}$. Immune profiles recently generated for 32 hip-replacement recipients revealed a link between the function of one immune cell type and the time to recovery from surgery ${ }^{18}$. After all, immune cells infiltrate every bodily system and have a role in virtually every disease. "We are only starting to understand the clinical applications," says Shen-Orr.

\section{Amanda B. Keener is a former news intern at Nature Medicine in New York and currently a freelance writer in Winston-Salem, North Carolina.}

1. Goodwin, K. et al. Vaccine 24, 1159-1169 (2006).

2. Rappuoli, R. and Aderem, A. Nature 473, 463-469 (2011).

3. Davis, M.M. \& Bjorkman, P.J. Nature 334, 395-402 (1988).

4. Schroeder H.W.Jr. Dev. Comp. Immunol. 30 119-135, (2006).

5. Freeman, J.D. et al. Genome Res. 19, 1817-1824 (2009).

6. Boyd, S.D. et al. Sci. Transl. Med. 1, 12ra23 (2009).

7. Kepler, T.B. et al. Cell Host Microbe 16, 304-313 (2014).

8. Jiang, N. et al. Sci. Transl. Med. 5, 171ra19 (2013).

9. Qi, Q. et al. Proc. Natl. Acad. Sci. 111, 13139-13144 (2014).

10. Wu, D. et al. Sci. Transl. Med. 4, 134ra63 (2012).

11. DeKosky, B.J. et al. Nat. Biotech. 31, 166-169 (2013).

12. DeKosky, B.J., et al. Nat. Med. doi:10.1038/nm.3743 (2014).

13. Han, A., Glanville, J., Hansmann, L. and Davis, M.M. Nat. Biotech. 32, 684-692 (2014)

14. Stern, J.N. et al. Sci. Transl. Med. 6, 248ra107 (2014).

15. van Heijst, J. W. et al. Nat. Med. 19, 372-377 (2013).

16. Muraro, P.A. et al. J. Clin. Invest. 124, 1168-1172 (2014).

17. Meyer, E.H. et al. Blood 121, 4955-5962 (2013).

18. Gaudillière, B. et al. Sci. Transl. Med. 6, $255 \mathrm{ra} 131$ (2014). 